\title{
Comparative effectiveness research and genomic personalized medicine
}

\section{"Comparative effectiveness research attempts to frame the delivery of healthcare by contrasting the benefits and harms of different diagnostic, therapeutic or preventative strategies in typical patients in an effort to define the most effective, safe and efficient interventions."}

\section{What is comparative effectiveness research?}

Although healthcare expenditures continue to rise rapidly in the USA, clinical measures of the health of the population have fallen behind many other developed countries. As shown in Figure 1, the average US healthcare expenditures have grown more rapidly over the past 30 years than in any other major industrialized country. Cancer treatments constitute a substantial proportion of these escalating costs, with novel biologic agents constituting an ever greater share [1]. Even more distressing is evidence that outcomes achieved from that healthcare spending, including life expectancy, are poor compared with health outcomes in other industrialized nations. (Figure 2) [2]. To address this challenging environment, it is essential to identify important diagnostic and therapeutic questions, and to acquire, evaluate and, where appropriate, synthesize all relevant information related to effectiveness, safety and overall value of comparative diagnostic, treatment and prevention strategies in order to properly inform important clinical and policy decisions. Comparative effectiveness research (CER) attempts to frame the delivery of healthcare by contrasting the benefits and harms of different diagnostic, therapeutic or preventative strategies in typical patients in an effort to define the most effective, safe and efficient interventions.

The Institute of Medicine (Washington, DC, USA) defines CER as [3]:

"...the generation and synthesis of evidence that compares the benefits and harms of alternative methods to prevent, diagnose, treat and monitor a clinical condition, or to improve the delivery of care. The purpose of comparative effectiveness research is to assist consumers, clinicians, purchasers and policy-makers to make informed decisions that will improve healthcare at both the individual and population levels."
Fundamental to this definition is the direct comparison of the effectiveness and safety of different interventions in 'typical' patients seen in everyday practice. Comparative results from prospective randomized controlled clinical trials (RCTs) or systematic reviews of such trials represent the 'gold standard' of CER. Since RCTs may be of poor quality, not available or sometimes even unethical and, at best, based on highly selected patients not representative of the general population, alternative sources of evidence are often utilized to guide the evaluation of new interventions and address the need for patients, clinicians and policy-makers to make critical decisions. The limitations of available evidence from RCTs have led to the exploration of additional sources, which, when applied with the same rigorous attention to study design, conduct, analysis and reporting, may provide reasonable, valid and generalizable estimates of comparative effectiveness, safety and costs (Box 1). Such approaches include carefully conducted cohort and population studies, as well as clinical simulations utilizing evidence from RCTs, meta-analyses, cohort and population studies to emulate realistic clinical scenarios reflecting the impact of an intervention on clinical effectiveness, toxicity, quality of life and costs. The ultimate goal of CER is to gather the totality of available evidence to address the comparative effectiveness, toxicity and overall value of established and emerging interventions.

\section{Comparative effectiveness of personalized medicine approaches}

Perhaps the greatest opportunity as well as challenge for CER relates to the evaluation of genomic personalized medicine approaches based on clinical, molecular and genomic biomarkers for identifying patients at increased risk for disease occurrence or recurrence as well
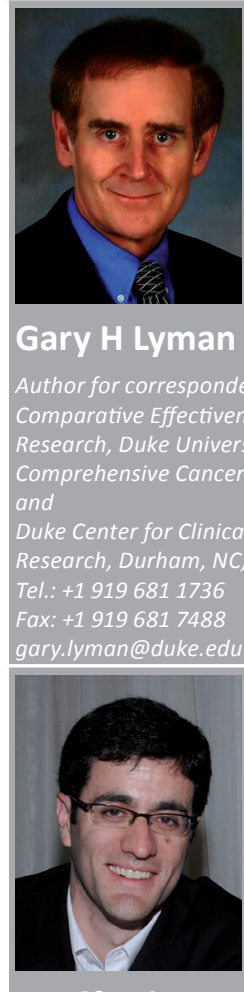

Bradford Hirsch

Division of Hematology/Oncology, Department of Medicine, Duke University, Durham, NC, USA 


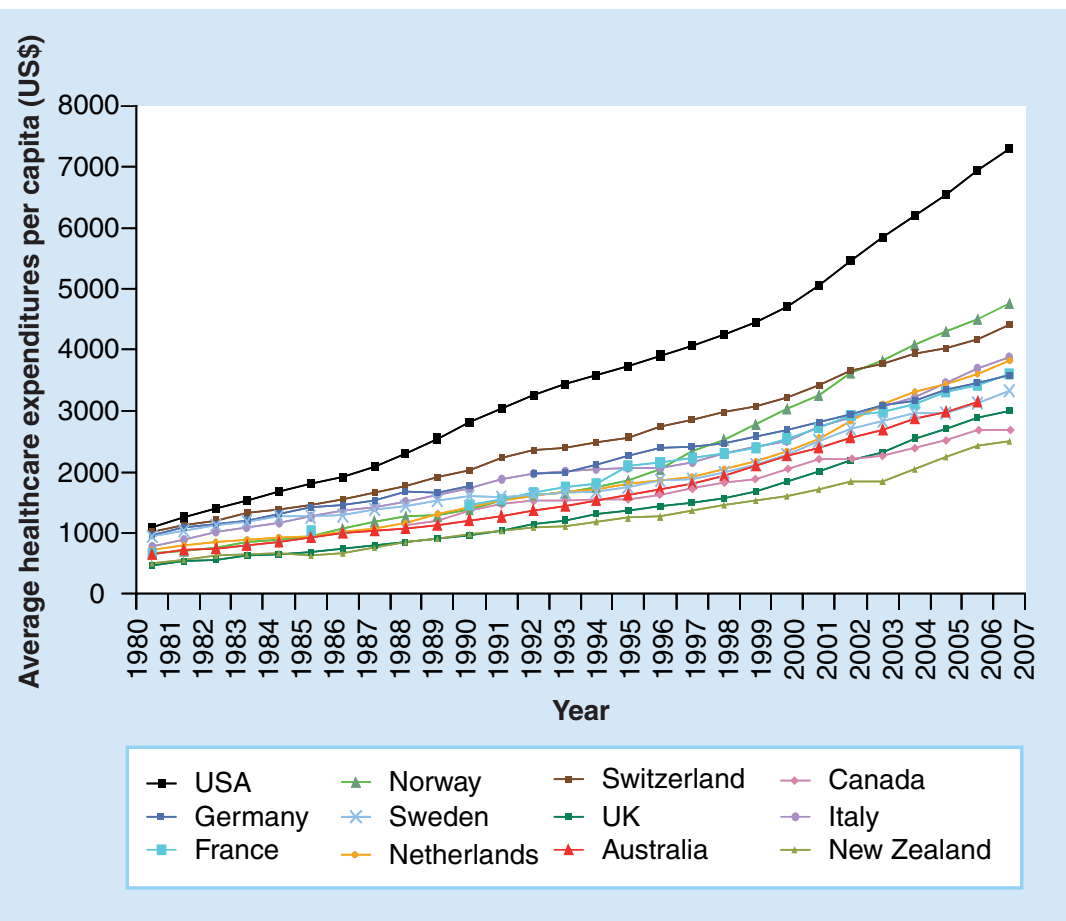

Figure 1. Annual healthcare expenditures for major industrialized nations. Data from [101]. somewhat muted the enthusiasm surrounding the rapid and accelerating pace of new diagnostic and therapeutic innovations, it has only confirmed the need for the deliberate and appropriate application of CER to such discoveries. Since treatment-related complications represent a major cause of morbidity and mortality in the general cancer population, targeted interventions are designed to minimize toxicity in patients unlikely to benefit, while maximizing treatment effectiveness and cost-effectiveness in those who will. The potential for more targeted, individualized or personalized interventions, tailoring treatments to patients at greatest risk and most likely to benefit, has fostered considerable enthusiasm among patients, clinicians and researchers. In oncology, such personalized strategies include the pharmacogenomics and pharmacogenetics of cancer therapies based on the molecular and genetic makeup of patients and their tumors. Targeted personalized interventions may have the potential for increasing the effectiveness of cancer therapies while reducing the frequency of treatment-related complications by identifying patients unlikely to benefit from an intervention where an alternative treatment or a research protocol may be considered. It has also not gone unnoticed that more selective and targeted use of new, expensive technologies may either reduce associated healthcare as greater benefit or harm with available treatments [4]. Dramatic advances in molecular biology and genetics have identified multiple potential targets for the diagnosis, treatment and prevention of cancer. While the relentless increase in healthcare expenditures has

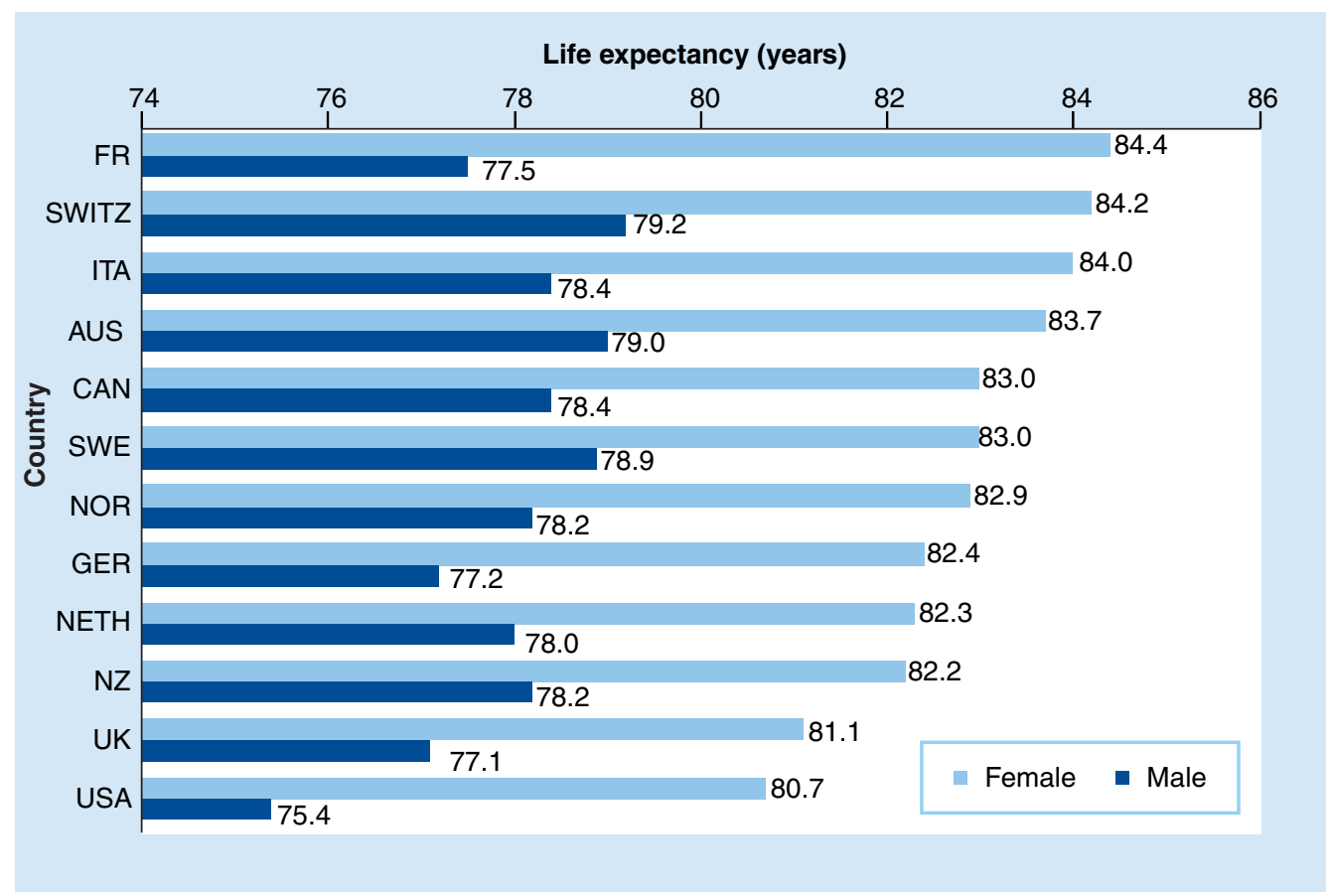

Figure 2. Representative estimated life expectancy from birth for major industrialized nations.

Data from [101]. 
expenditures or optimize the effectiveness gained with the resources applied in the care of patients with cancer.

\section{“...targeted interventions are designed to minimize toxicity in patients unlikely to benefit while maximizing treatment effectiveness and cost-effectiveness in those who will."}

As previously discussed, the highest level of evidence for the CER studies of such approaches would be in well-designed, prospective RCTs comparing guided or targeted intervention to concurrent unguided intervention. Alternative CER studies utilizing well-matched concurrent control subjects but otherwise rigorous methods may also be considered [5]. Such CER studies should be distinguished from association studies utilized in the development (Phase I) or validation (Phase II) of prognostic or predictive models (Box 2) [5]. Retrospective analyses of prospective clinical trials with archived tissue samples permitting evaluation of potentially important genetic and molecular markers in patients with valid outcome data have been effectively pursued $[6,7]$.

Nevertheless, genetic and molecular biomarkers must be evaluated rigorously like any diagnostic, prognostic or predictive assay, considering both test performance characteristics as well as the underlying patient population. Studies of gene-profile assays to predict disease recurrence in patients with early-stage breast cancer were systematically reviewed demonstrating large variation in predictive performance [8]. CER studies of gene-expression assays based on clinical decision simulation studies have been conducted incorporating measures of prognostic and predictive validity data from clinical trials [9,10]. Utilizing Monte Carlo simulation and Markov modeling, these studies have demonstrated the favorable cost-effectiveness of adjunctive therapy based on the results of the assay compared with other standard therapeutic strategies (Figure 3). Recommendations have recently been put forward for the complete and transparent reporting of prognostic and predictive biomarker studies in oncology [11].

\section{Codevelopment of targeted therapies \& predictive biomarkers}

While biomarker assays will be developed for most novel agents, thereby allowing better patient selection for targeted intervention in those most likely to benefit while avoiding

\section{Box 1. Toolbox for comparative effectiveness research.}

\section{- Randomized controlled trials}

- Systematic reviews and meta-analyses

- Other comparative clinical trials

- Population studies, including registries and administrative and claims data

Prognostic and predictive association studies

- Quality-of-life studies, including patient-reported outcomes

- Clinical decision-making models, including cost-effectiveness and cost-utility analyses

Adapted with permission from [13].

exposure and complications in those unlikely to respond, development of targeted agents prior to assay development represents an emerging challenge for CER. Nowhere is this better illustrated than with the recent availability of EGF receptor inhibitors with subsequent development of assays for mutations of the KRAS oncogene. The predictive accuracy of these assays was validated only during or following completion of the large registration clinical trials [12]. When conducting large RCTs of targeted agents, the potential need to validate such prognostic and predictive biomarkers in the future should be anticipated by archiving tissue and blood. Box 3 summarizes recent recommendations for the appropriate codevelopment of biomarkers in trials of targeted agents in order to avoid dependence upon exploratory studies for clinical validation or regulatory approval [13]. Large confirmatory trials of targeted biologic therapies should be encouraged if not required to archive tissue samples on all subjects in order to facilitate future validation of potential biomarkers. It is also critical that individual patient data be shared across clinical trials for future CER based on pooled analyses of biomarker assay performance information in different populations [14].

\section{"development of targeted agents prior to assay development represents an emerging challenge for comparative effectiveness research."}

\section{Conclusion}

Comparative effectiveness research applied to genomic personalized medicine approaches should not be considered as a method for avoiding

\section{Box 2. Types of prognostic and predictive studies.}

\footnotetext{
- Phase I: exploratory association studies (derivation)

- Phase II: confirmatory association studies (validation)

- Phase III: comparative effectiveness studies

- Randomized: guided vs unguided controls

- Nonrandomized: guided vs unguided concurrent controls

- Nonrandomized: guided vs historical controls
} 


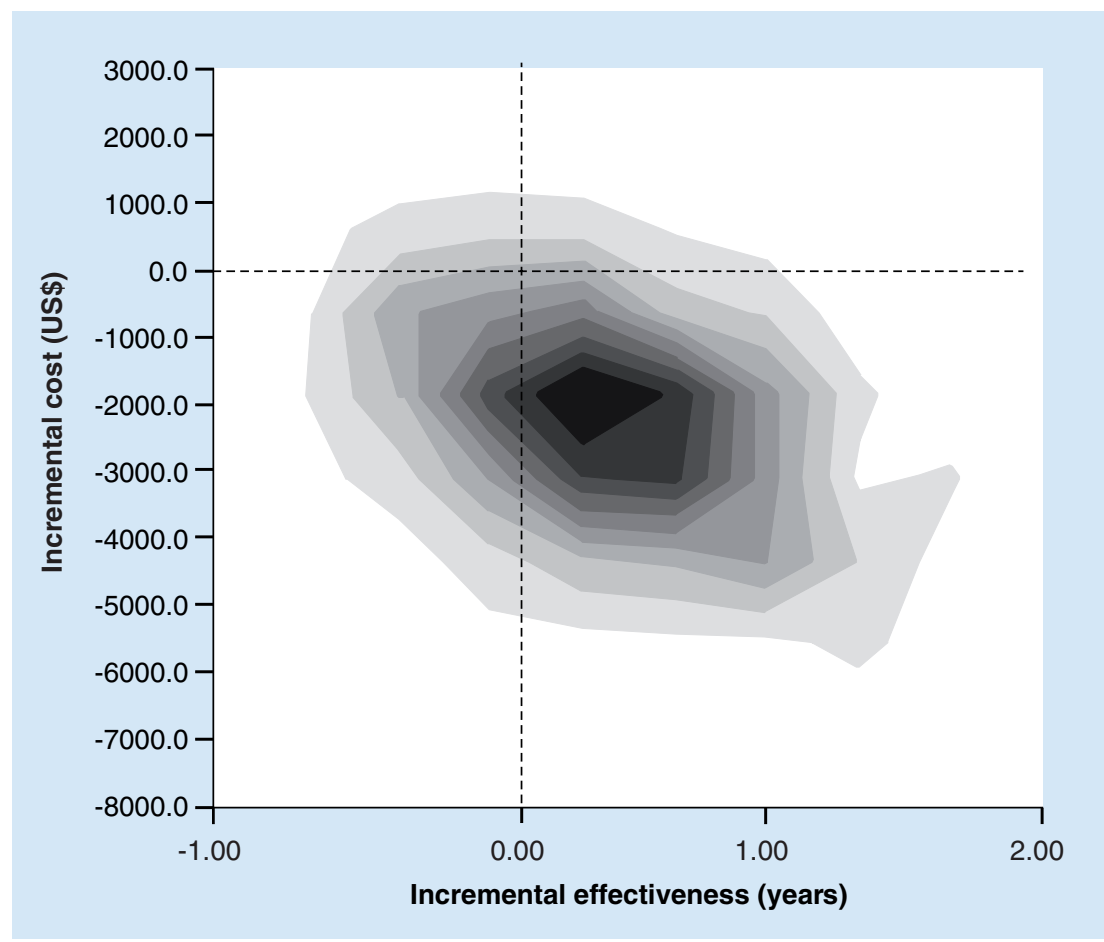

Figure 3. Isocontours from Monte Carlo simulation of incremental cost and effectiveness for 21-gene reverse transcriptase PCR gene-expression assays compared with standard treatment in patients with early-stage breast cancer.

Adapted with permission from [101].

the effort and cost of conducting a full evaluation of all available evidence on new technologies, such as those for achieving genomic personalized medicine. RCTs remain the 'gold standard' of CER and an excellent source of information to inform population and decision-modeling studies. However, the exponential growth and complexity of data regarding genomic biomarkers for more individualized targeting of established as well as emerging therapies represents an enormous challenge for clinicians, researchers and policy-makers in the setting of rapidly rising healthcare costs. Identifying patients at increased or decreased risk as well as those most likely to benefit or to be harmed by potentially toxic and costly treatments represents a compelling if not required direction for improving healthcare outcomes while providing the greatest value for patients and society when faced with finite resources. CER is, therefore, essential for systematically identifying and summarizing the totality of evidence on the effectiveness, safety and value of competing strategies, including results from RCTs and meta-analyses, population studies and clinical simulation studies, in order to inform and provide patients, providers and policy-makers valid recommendations for the utilization of genomic personalized molecular and genetic approaches.

\section{Financial \& competing interests disclosure \\ Dr Gary Lyman is supported by grants from the National Cancer Institute (RC2CA148041-01) and the National Heart, Lung and Blood Institute (1R01HL095109-01). The authors have no other relevant affliations or financial involve- ment with any organization or entity with a financial interest in or financial conflict with the subject matter or materials discussed in the manuscript apart from those disclosed. \\ No writing assistance was utilized in the production of this manuscript.}

\section{Box 3. Recommendations for the codevelopment of targeted therapies and predictive biomarkers.}

- Source studies should be well-designed, large, randomized trials with appropriate control groups and clinically relevant end points of efficacy and safety

- Biomarkers should be based on a biologically plausible rationale and have good test performance and reproducibility

- Biomarker results should be available on a majority of subjects with a detailed account of the reasons for unavailable samples or results

- Biomarker subgroups and planned analysis should be prespecified

- There should be adequate power to establish with confidence any differential treatment effect in subgroups based on the biomarker

- Biomarker measurement should be blinded to the treatment group assignment and study outcomes

- Appropriate adjustment for multiple testing should be carried out

- Formal testing of any drug-biomarker interaction is needed

- Results should be adjusted for all known prognostic/predictive factors

- Consistent findings related to the biomarker should be observed in at least two large trials

Adapted with permission from [13]

\section{Bibliography}

1 Meropol NJ, Schulman KA: Cost of cancer care: issues and implications. J. Clin. Oncol. 25, 180-186 (2007).

2 Davis K: Slowing the growth of healthcare costs - learning from international experience. N. Engl. J. Med. 359, 1751-1755 (2008).
3 Sox HC, Greenfield S: Comparative effectiveness research: a report from the Institute of Medicine. Ann. Intern. Med. 151, 203-205 (2009).

4 Riley RD, Sauerbrei W, Altman DG: Prognostic markers in cancer: the evolution of evidence from single studies to meta-analysis, and beyond. Br. J. Cancer 100, 1219-1229 (2009).
5 Altman DG, Lyman GH: Methodological challenges in the evaluation of prognostic factors in breast cancer. Breast Cancer Res. Treat. 52, 289-303 (1998).

6 Paik S, Shak S, Tang G et al.: A multigene assay to predict recurrence of tamoxifen-treated, node-negative breast cancer. N. Engl. J. Med. 351, 2817-2826 (2004). 
7 Paik S, Tang G, Shak S et al.: Gene expression and benefit of chemotherapy in women with node-negative, estrogen receptor-positive breast cancer. J. Clin. Oncol. 24, 3726-3734 (2006).

Lyman GH, Kuderer NM: Gene expression profile assays as predictors of recurrence-free survival in early-stage breast cancer: a metaanalysis. Clin. Breast Cancer 7, 372-379 (2006).

9 Hornberger J, Cosler LE, Lyman GH: Economic analysis of targeting chemotherapy using a 21-gene RT-PCR assay in lymph-nodenegative, estrogen-receptor-positive, early-stage breast cancer. Am. J. Manag. Care 11, 313-324 (2005).

10 Lyman GH, Cosler LE, Kuderer NM et al.: Impact of a 21-gene RT-PCR assay on treatment decisions in early-stage breast cancer: an economic analysis based on prognostic and predictive validation studies. Cancer 109, 1011-1018 (2007).

11 McShane LM, Altman DG, Sauerbrei W et al:: Reporting recommendations for tumor marker prognostic studies (REMARK). J. Natl Cancer Inst. 97, 1180-1184 (2005).

12 Allegra CJ, Jessup JM, Somerfield MR et al.: American Society of Clinical Oncology provisional clinical opinion: testing for $K R A S$ gene mutations in patients with metastatic colorectal carcinoma to predict response to anti-epidermal growth factor receptor monoclonal antibody therapy. J. Clin. Oncol. 27, 2091-2096 (2009).
13 Lyman GH: Comparative effectiveness research in oncology: the need for clarity, transparency and vision. Cancer Invest. 27, 593-597 (2009).

14 Teutsch SM, Bradley LA, Palomaki GE et al:: The Evaluation of Genomic Applications in Practice and Prevention (EGAPP) Initiative: methods of the EGAPP Working Group. Genet. Med. 11, 3-14 (2009).

\section{Website}

101 Organisation for Economic Co-operation and Development (OECD) Health Data www.oecd.org 